\title{
ASYMPTOTIC SOLUTIONS OF INTEGRAL EQUATIONS WITH CONVOLUTION KERNELS
}

\author{
by V. HUTSON \\ (Received 1st February 1963)
}

\section{Summary}

The equations considered are Fredholm integral equations of the second kind with regular kernels, whose argument depends only on the difference of the variables. Approximate solutions are sought for a given finite range of the eigenvalues, and for large values of the range of integration. Certain special conditions are imposed on the general form of the Fourier transforms of the kernel. Then it is shown that approximate solutions may be obtained in terms of the solutions of the corresponding (singular) Wiener-Hopf equations. Approximations to the eigenvalues are also found. It is shown that the eigenfunctions are unique, and that except possibly near the end points of the range, the solutions are of trigonometric type with the zeros of successive solutions interlacing.

\section{Introduction}

The equations considered are the Fredholm integral equations of the second kind

$$
\begin{array}{ll}
f(x)=\lambda_{n} \int_{0}^{a} k(x-t) f(t) d t & (0 \leqq x \leqq a), \\
f(x)=\lambda \int_{0}^{a} k(x-t) f(t) d t+g(x) & (0 \leqq x \leqq a) .
\end{array}
$$

The solution of (1.1) is assumed to satisfy a condition similar to a normalisation condition

$$
\int_{0}^{a} f^{2}(x) d x=a .
$$

The kernel is defined in $(-\infty, \infty)$ and is taken to be regular, independent of $a$, even, and exponentially small at infinity. The unknown functions $f$ depend on $\lambda$ and $a$, and approximate solutions will be obtained when $\lambda$ lies in any given finite range and $a$ is large. Attention is focussed particularly on (1.1).

The Fredholm equation with a regular kernel has been studied extensively, see for example (3) or (11). It is known that the eigenvalues, that is the values $\lambda_{n}$ for which (1.1) has solutions (eigenfunctions), form a discrete set and that 
each eigenvalue is of finite index, the index being defined as the number of linearly independent eigenfunctions corresponding to each eigenvalue.

Even if the kernel depends only on the difference of the variables, it is difficult to find solutions or to obtain any general information about the general form of the solutions or about the spectrum, unless the kernel is of specially simple form.

Latter (7) obtained approximate solutions of these equations for large $a$ by Fourier transform methods. He shows how to find approximate solutions, say $f_{1}(x)$, such that for sufficiently large $a$

$$
f(x)=f_{1}(x)+O\left(e^{-x}+e^{a-x}\right) .
$$

This approximation is thus not uniformly valid in $(0, a)$ in the sense of the present results (1.6). Latter derived his solution from an approximation to an infinite system of linear algebraic equations, but did not prove the validity of his approximating process and did not study the uniqueness of the solutions. However, the present investigation is based on Latter's method.

Widom (12) showed that if the Fourier transform $K$ of the kernel is decreasing near the origin and $d^{2} K(0) / d w^{2} \neq 0$ then the eigenvalues of (1.1) are given by

$$
\lambda_{n}^{-1}=K(0)+\frac{1}{2}\left[\pi a^{-1}(n+1)\right]^{2} d^{2} K(0) / d w^{2}\left[1+O\left(a^{-1}\right)\right]
$$

for fixed $n$ as $a$ approaches infinity. Widom (13) also gives a method for finding the eigenfunctions. (In this paper the restriction $d^{2} K(0) / d w^{2} \neq 0$ is removed, but in the present investigation the condition is assumed to hold.) This expression for the eigenfunctions is however not uniformly valid throughout $(0, a)$ in the sense of the present result (1.6). Also both results only hold for fixed $n$ as $a$ becomes large, and as can be seen from (1.5), this implies that the range of values of $\lambda$ approaches zero as $a$ approaches infinity. In the present investigation this restriction is not necessary.

The approximate solutions of both these authors are not accurate near the end points of the range. In fact near these points the error may be as large as $O(1)$ for large $a$. Carrier (1) suggested that solutions of equations (1.1) and (1.2) may be found in terms of the solutions of the related Wiener-Hopf equations (4.1) and (4.2). Carrier did not give conditions under which this hypothesis should hold. The present investigation obtains appropriate conditions and shows that if they hold, Carrier's hypothesis is correct.

The main purpose of the present investigation is to examine (1.1), and to show that if the Fourier transform $K$ of the kernel is a decreasing function of $w$ ( $w$ positive) and $d^{2} K(0) / d w^{2} \neq 0$, then an approximate solution can be found in terms of $\phi$ the solution of the corresponding Wiener-Hopf equation (4.1) with an error which approaches zero as $a$ approaches infinity. That is, if $\lambda$ lies in any given finite range, $\lambda_{0}<\lambda<\lambda_{1}$ say, then

$$
\left.\begin{array}{rlrl}
f(x) & =\phi(x)+O\left(e^{-a \tau_{1} / 2}\right) & & \left(0 \leqq x \leqq \frac{1}{2} a\right), \\
& =\phi(a-x)+O\left(e^{-a \tau_{1} / 2}\right) & & \left(\frac{1}{2} a \leqq x \leqq a\right),
\end{array}\right\}
$$


for any $a$ greater than $a_{0}$, where $a_{0}, \tau_{1}$ are positive constants dependent only on $\lambda_{1}$. An equation for the eigenvalues is also found, and it is shown that they are of index unity. If, however, the Fourier transform of the kernel is not decreasing, then the solutions of (1.1) are not necessarily of index unity, and the situation is necessarily more complex. The present investigation does not cover this more general case, although a remark is made about it in Section 7. However, the methods used here could probably be extended to cover this case.

It is also shown here that for large $a$ equation (1.1) bears a resemblance to a Sturm-Liouville system. First the eigenvalues are of index unity. Also, by using the asymptotic formula (4.8) for the Wiener-Hopf equation, it is shown that in the region away from the end points of the range, the solutions are oscillatory and the zeros of successive solutions interlace (indeed the solutions are approximately trigonometric). This is discussed more fully in Section 7.

It is also shown in Section 8 , that for $\lambda<\lambda_{0}$ (the smallest eigenvalue of (1.1)), (1.2) can be solved approximately in terms of the resolvent kernel of the corresponding Wiener-Hopf equation (4.2).

\section{Notation and conditions on the kernel}

It is assumed that $k(x)$ is given for all real $x, f$ is then defined outside $(0, a)$, by requiring (1.1) and (1.2) to hold for all $x$. Take

$$
\begin{aligned}
& K(w)=\int_{-\infty}^{\infty} e^{i x w} k(x) d x, \quad F_{a}(w)=\int_{0}^{a} e^{i x w} f(x) d x, \\
& F_{+}(w)=\int_{a}^{\infty} e^{i x w} f(x) d x, \quad F_{-}(w)=\int_{-\infty}^{0} e^{i x w} f(x) d x, \\
& G_{a}(w)=\int_{0}^{a} e^{i x w} g(x) d x .
\end{aligned}
$$

It follows that, if $f(x)= \pm f(a-x)$, that is, if $f(x)$ is symmetric or antisymmetric about $\frac{1}{2} a$, then

$$
F_{+}(w)= \pm e^{i w a} F_{-}(-w) .
$$

It is assumed that the kernel and its Fourier transform satisfy the following conditions.

Condition $A . \quad k$ is a real bounded, continuous, even function. Also there exists a positive number $\tau$ such that $k(x) \exp (s x)$ belongs to $L(0, \infty)$ and $L^{2}(0, \infty)$ for $s \leqq \tau$. The derivative $d k / d x$ is bounded and continuous except for at most a finite number of discontinuities. Also the integral

$$
\int_{x}^{\infty}[d k(x) / d x]^{2} d x
$$

exists and considered as a function of $x$ belongs to $L(0, \infty)$. 
Condition $B$. For $w$ real and positive $K(w)$ is a strictly decreasing function.

Condition $C . d^{2} K(0) / d w^{2} \neq 0$.

It is easy to deduce from $A$ and $B$ that $K$ is positive for real $w$.

\section{Deductions from the general theory}

A few simple results are noted; these results either come directly from the general theory (3) and (11) or are simply deduced from it.

(a) The kernel is positive definite (in the sense defined below), and so (3) has only positive eigenvalues.

(b) Any eigenfunction is either symmetric or antisymmetric, or may be expressed as the sum of symmetric and antisymmetric eigenfunctions.

(c) If for any eigenvalue there are two symmetric eigenfunctions there must also be an antisymmetric eigenfunction, with a similar result for antisymmetric functions.

(d) There exists a positive constant $c$ independent of $a$ such that for sufficiently large $a$ and all $n$,

$$
\lambda_{n} \geqq K^{-1}(0)+c a^{-2} \text {. }
$$

(e) The solution of (1.2) satisfies the relation

$$
\int_{0}^{a} f^{2}(x) d x \leqq\left(1-\lambda \lambda_{0}^{-1}\right)^{-2} \int_{0}^{a} g^{2}(x) d x .
$$

A kernel is defined to be positive definite if for all integrable non-null functions $f$

$$
J(f, f)=\int_{0}^{a} \int_{0}^{a} k(s-t) f(s) f(t) d s d t>0
$$

But

$$
J(f, f)=\frac{1}{2 \pi} \int_{-\infty}^{\infty} K(w)\left|F_{a}(w)\right|^{2} d w
$$

which is clearly positive as $K(w)$ is positive.

(b) is clearly a consequence of the evenness of the kernel. To prove (c) form an eigenfunction $f_{3}=C f_{1}+D f_{2}$ where $f_{1}$ and $f_{2}$ are symmetric eigenfunctions, and $C, D$ are chosen so that $f_{3}(0)=f_{3}(a)=0$. Then an integration by parts shows that the derivative of $f_{3}$, an anti-symmetric function, is also an eigenfunction.

(d) is proved by Widom (12).

To prove $(e)$ it is first noted that

$$
\lambda_{0}=\inf \left\{J^{-1}(f, f) \int_{0}^{a} f^{2}(x) d x\right\} .
$$


Multiplying (1.2) by $f$, integrating and using Schwartz's inequality, we have

$$
\int_{0}^{a} f^{2}(x) d x \leqq\left\{\int_{0}^{a} f^{2}(x) d x \int_{0}^{a} g^{2}(x) d x\right\}^{\frac{1}{2}}+\lambda \int_{0}^{a} \int_{0}^{a} k(x-t) f(x) f(t) d x d t .
$$

Relation (3.2) follows from (3.3).

\section{The Wiener-Hopf equations}

It is well known that the solutions of the Wiener-Hopf integral equations

$$
\begin{array}{ll}
\phi(x)=\lambda \int_{0}^{\infty} k(x-t) \phi(t) d t & \left(\lambda>K^{-1}(0)\right), \\
\phi(x)=\lambda \int_{0}^{\infty} k(x-t) \phi(t) d t+g(x) & \left(\lambda<K^{-1}(0)\right),
\end{array}
$$

may be reduced to quadratures. These equations are not regular and their eigenvalues do not form a discrete set. Accounts of the method of solution may be found in (2), (4), (6), (8), (9), (10). Approximate methods are also given in (2) and (8). Even when these methods are difficult, it is possible to find an asymptotic solution of (4.1) for large $x$ from a knowledge of the Fourier transform of the kernel alone; the eigenfunctions are found to be of trigonometric type (4.8).

The following results are standard and are taken mainly from (9) with the requisite changes of notation to meet the present special case. Expressions as quadratures are given there for $K_{+}$and $K_{-}$defined below.

Condition $B$ implies that for fixed $\lambda>K^{-1}(0)$ there are exactly two real roots, $\pm \mu$ say, of $1-\lambda K(w)=0$. Thus $\lambda=K^{-1}(\mu)$. Also it is known that $|K(w)|$ approaches zero uniformly in the strip $|\Im \mathfrak{w}|<\tau$. This together with conditions $B$ and $C$ implies that, for $\mu$ in some fixed range $0<\mu<\mu_{1}$ say, there is a constant $\tau_{1}\left(0<\tau_{1}<\tau\right)$ independent of $\mu$ such that there are only the two real zeros in the strip $|\mathfrak{I} w| \leqq \tau_{1}$. It is always assumed when dealing with (4.1) that $\mu$ lies in the given range.

The solutions of (4.1) depend on the following factorisation theorem. In the strip $\left|\Im_{w}\right| \leqq \tau_{1}$, it is possible to write

$$
1-\lambda K(w)=\frac{K_{-}(w)}{K_{+}(w)}\left(w^{2}-\mu^{2}\right),
$$

where $K_{-}$is regular and free from zeros for $\mathfrak{I} w<\tau_{1}$, and $K_{+}$has the same property for $\mathfrak{I} w>-\tau_{1}$. The moduli $\left|w K_{-}(w)\right|$ and $\left|w^{-1} K_{+}(w)\right|$ lie for sufficiently large $w$ between positive bounds in their respective half planes of regularity, these bounds being dependent only on $\mu_{1}$. The functions $K_{+}$ and $K_{-}$satisfy the relations

$$
\begin{aligned}
K_{ \pm}(w) & =-K_{\mp}^{-1}(-w), \\
K_{ \pm}(-\bar{w}) & =-\overline{K_{ \pm}(w)} \ldots \ldots
\end{aligned}
$$


These functions depend on $\mu$ and will be written $K_{+}(w ; \mu), K_{-}(w ; \mu)$ when this dependence needs to be emphasised. The derivative $\partial K_{ \pm}(\mu ; \mu) / \partial \mu$ is a continuous function of $\mu$.

If $\lambda<K^{-1}(0)$ there will be no real zeros in a strip $\mathfrak{I} w<\sigma$ say. The factorisation then becomes

$$
1-\lambda K(w)=K_{-}^{1}(w) / K_{+}^{1}(w) .
$$

Equation (4.1) has just one solution (except for multiplication by a constant) which has a bound independent of $x$.

$$
\phi(x)=B^{-1} \lim _{A \rightarrow \infty} \frac{1}{2 \pi} \int_{i \tau_{1}-A}^{i \tau_{1}+A} e^{-i w x} K_{+}(w)\left(w^{2}-\mu^{2}\right)^{-1} d w .
$$

Thus the integral must be interpreted in the Plancherel sense. That is, as $A$ approaches infinity the integral tends in mean square to a function $\phi$ such that $\phi \exp (-\tau x)$ belongs to $L^{2}(0, \infty)$. The number $B$ has been chosen so that

$$
2 a^{-1} \int_{0}^{a / 2} \phi^{2}(x) d x=1
$$

Further it is possible to show (9, Theorem 16) that $\phi$ is a bounded continuous function. Indeed $|\phi|$ has a bound independent of both $\mu$ and $x$. Also, for the relevant range $\mu>c a^{-1}, B=O(a)$.

It can easily be shown (9) that

$$
B \phi(x)=\Re \mu^{-1} K_{+}(\mu) e^{-i \mu x}+O\left(e^{-\tau_{1} x}\right)(x \rightarrow \infty) .
$$

In (4.2) it is assumed that $|g|=O(\exp (-\beta x))$ for some positive $\beta$. Equation (4.2) has just one solution which is bounded at infinity.

$$
\text { , } \phi(x)=\lim _{A \rightarrow \infty} \frac{1}{2 \pi} \int_{-A}^{A} e^{-i x w} K_{+}^{1}(w) G_{+}(w) d w,
$$

where

$$
G_{+}(w)=\frac{G(w)}{K_{-}^{1}(w)}+\frac{1}{2 \pi i} \int_{i \sigma-\infty}^{i \sigma+\infty} \frac{G\left(w^{\prime}\right) d w^{\prime}}{K_{-}^{1}\left(w^{\prime}\right)\left(w^{\prime}-w\right)}(\mathfrak{I} w<\sigma)
$$

\section{Approximate solution of the homogeneous equation (1.1)}

In this section it will be proved that for a given finite range of values of $\lambda$ (and so of $\mu$ since $\lambda=K^{-1}(\mu)$ ), every solution of (1.1) is given approximately for $a$ large enough by equation (5.15) and the relation between $\mu$ and $a$ by equation (6.1). In Appendix 2 it will be shown conversely that every $\mu$ (lying in the given range) that satisfies (6.1) and (d) of Section 3 provides a solution (1.1). A further discussion of the spectrum is given in Section 6.

The approximation is based on an approximate method of Latter (7) described later in this section. It was pointed out in the introduction that 
Latter's solution is not uniformly valid, and that the uniqueness and convergence were not discussed. The present investigation makes certain assumptions about the kernel (conditions $B$ and $C$ ) and proves rigorously that the solutions of equation (1.1) are closely related to the solutions of the corresponding Wiener-Hopf equation (4.1) with an error which is uniformly small for large $a$. The uniqueness is discussed in Section 6 .

Choose a positive constant $\tau_{2}<\tau_{1}$. Let $C_{1}, C_{2}, C_{2}^{\prime}, C_{3}$ be contours lying in the strip $\left|\Im_{w}\right| \leqq \tau_{2}$. Let $C_{1}$ be the line joining $i \tau_{2}-\infty$ to $i \tau_{2}+\infty$. Let $C_{2}, C_{2}^{\prime}$ be contours joining $-\infty$ to $\infty$ and lying above the point $w=-\mu$ and below $w=\mu$. Let $C_{3}$ be the line joining $-i \tau_{2}-\infty$ to $-i \tau_{2}+\infty$.

Latter showed that the problem could be formulated as follows in terms of Fourier transforms. The method is similar to the Wiener-Hopf method, but does not in this case lead to an explicit solution.

In the strip of regularity,

$$
-F_{a}(w)=\frac{F_{+}(w)}{\left(w^{2}-\mu^{2}\right)} \frac{K_{+}(w)}{K_{-}(w)}+\frac{F_{-}(w)}{\left(w^{2}-\mu^{2}\right)} \frac{K_{+}(w)}{K_{-}(w)}
$$

The functions $F_{+}$and $F_{-}$are the solutions of the simultaneous integral equations

$$
\begin{gathered}
\frac{F_{-}(w)}{(w-\mu) K_{-}(w)}=\frac{1}{2 \pi i} \int_{C_{2}} \frac{d w^{\prime}}{w^{\prime}-w} \frac{F_{+}\left(w^{\prime}\right)}{\left(w^{\prime}-\mu\right) K_{-}\left(w^{\prime}\right)}, \ldots \ldots \\
\frac{F_{+}(w) K_{+}(w) e^{-i a w}}{(w+\mu)}=-\frac{1}{2 \pi i} \int_{C_{2}^{\prime}} \frac{d w^{\prime}}{w^{\prime}-w} \frac{F_{-}\left(w^{\prime}\right) K_{+}\left(w^{\prime}\right) e^{-i a w^{\prime}}}{\left(w^{\prime}+\mu\right)},
\end{gathered}
$$

where the two integrals are evaluated for $w$ below and above $C_{2}, C_{2}^{\prime}$ respectively. The solution is then found from the Fourier inversion formula

$$
f(x)=\frac{1}{2 \pi} \int_{C_{2}} F_{a}(w) e^{-i x w} d w .
$$

Latter obtained approximate solutions of (5.2) and (5.3) in the following manner. In the first equation the contour is raised to sweep across a finite number of the poles of the integrand, the remaining integral being ignored. In the second the contour is lowered. A system of linear algebraic equations is then obtained by letting $w$ approach in turn the poles of $(w-\mu)^{-1} K_{-}^{-1}(w)$ and $(w+\mu)^{-1} K_{+}(w)$ respectively. Latter then obtained the solution by evaluating (5.4) by allowing the contour to sweep across some finite number of the poles of the approximately calculated integrand. The present investigation shows that, if conditions $B$ and $C$ hold, it is possible to obtain a uniformly valid approximation by considering only two of the poles in setting up the algebraic equations, but by an evaluation of the integral in (5.4) with an error which is exponentially small.

In (5.2), $C_{2}$ is changed to $C_{1}$ (crossing the pole at $\mu$ ) and, in (5.3), $C_{2}^{\prime}$ is 
changed to $C_{3}$ (crossing the pole at $-\mu$ ). Then

$$
\begin{aligned}
\frac{F_{-}(w)}{(w-\mu) K_{-}(w)} & =-\frac{F_{+}(\mu)}{(w-\mu) K_{-}(\mu)}+R(w), \ldots \\
\frac{F_{+}(w) K_{+}(w) e^{-i a w}}{w+\mu} & =-\frac{F_{-}(-\mu) K_{+}(-\mu)}{w+\mu}-S(w) .
\end{aligned}
$$

Expressions for $R$ and $S$ are given in Appendix 1, equation (A4).

Now let $w$ approach $\pm \mu$ in turn. Then

$$
\begin{array}{r}
\frac{F_{-}(-\mu)}{K_{-}(-\mu)}+\frac{F_{+}(\mu)}{K_{-}(\mu)}=2 \mu R(-\mu), \ldots \ldots \ldots \ldots \\
F_{+}(\mu) K_{+}(\mu)+F_{-}(-\mu) K_{+}(-\mu) e^{2 i a \mu}=-2 \mu S(\mu) e^{i a \mu} .
\end{array}
$$

It may be seen from (2.1) that for symmetric and antisymmetric eigenfunctions these equations are identical. Thus for symmetric and antisymmetric eigenfunctions respectively

$$
F_{+}(\mu)\left[K_{+}(-\mu) \pm e^{-i \mu a} K_{+}(\mu)\right]=-2 \mu R(-\mu) .
$$

It may be seen from (A5) that the right-hand side of this equation is exponentially small for large $a$. However, for the moment nothing can be said about the relation between $\mu$ and $a$, although it will later be proved that this bracket is small for large $a$.

The approximate solution of (1.1) is now obtained by substituting (5.5) and (5.6) into (5.4). Equations (2.1) and (4.4) have been used to simplify the equation. Then

$$
\begin{array}{r}
2 \pi f(x)=-F_{+}(\mu) K_{+}(-\mu) \int_{C_{2}} d w\left[\mp e^{i w(a-x)} \frac{1}{\left(w^{2}-\mu^{2}\right) K_{-}(w)}+e^{-i x w} \frac{K_{+}(w)}{w^{2}-\mu^{2}}\right] \\
-\int_{C_{2}} e^{-i x w} \frac{R(w) K_{+}(w) d w}{w+\mu}+\int_{C_{2}} e^{i w(a-x)} \frac{S(w) d w}{(w-\mu) K_{-}(w)}, \ldots \ldots(5.10)
\end{array}
$$

where the two signs refer to symmetric and antisymmetric eigenfunctions respectively.

It will now be shown that the last two terms in this expression are small in mean square, and it will be proved later that they are uniformly small. The first is then shown to be related to the Wiener-Hopf solution.

After a change of the line of integration, it is easily seen that the third term is

$$
e^{i x \mu} R(-\mu) K_{+}(-\mu)+\int_{C_{3}} e^{-i x w} R(w) K_{+}(w) \frac{d w}{(w+\mu)} .
$$

Let this integral be $h(x)$. By (A5) the integral belongs to $L^{2}(-\infty, \infty)$ and is also exponentially small. Hence, by Plancherel's theorem and by (A5),

$$
\begin{aligned}
\int_{-\infty}^{\infty} h^{2}(x) d x & =e^{-2 x \tau_{2}} \int_{-i \tau_{2}-\infty}^{i \tau_{2}+\infty}\left[\frac{R(w) K_{+}(w)}{w+\mu}\right]^{2} d w \\
& =O\left(a e^{-2 a \tau_{1}}\right) .
\end{aligned}
$$

for sufficiently large $a$. The same result applies for the last term. 
Therefore, by a change of contour,

$$
\begin{aligned}
2 \pi f(x)=-F_{+}(\mu) K_{+}(-\mu)\left\{B \phi(x)-\frac{e^{-i \mu x}}{2 \mu}\right. & {\left[K_{+}(\mu) \pm e^{i a \mu} K_{+}(-\mu)\right] } \\
\mp & \left.\int_{C_{1}} e^{i w(a-x)} \frac{d w}{\left(w^{2}-\mu^{2}\right) K_{-}(w)}\right\}+\varepsilon(x),
\end{aligned}
$$

where $\phi$ is the solution of the Wiener-Hopf equation (4.7), and $\varepsilon$ is an error term which is small in mean square. The integral may be shown by Plancherel's theorem to be of the form $m(x) \exp \left[-(a-x) \tau_{1}\right]$ for $x \leqq \frac{1}{2} a$, where the square integral of $m(x)$ is bounded in $a$. A similar result may be obtained for $a \geqq x \geqq \frac{1}{2} a$.

Now, by (d) of Section 3, $\mu>c a^{-1}$ for some constant $c$, and as stated in Section $4, B$ is then $O(a)$ for this range of values of $\mu$. Therefore, because of the factor $F_{+}(\mu)$ multiplying the solution, it is now obvious from equations (5.9) and (A5) that, unless

$$
K_{+}(-\mu) \pm e^{-i \mu a} K_{+}(\mu)=O\left(a^{\frac{3}{2}} e^{-a \tau_{1}}\right),
$$

$f(x)$ will be too small to satisfy (1.3) for sufficiently large $a$. (5.13) is an equation for the relation between $\mu$ and $a$ and determines the eigenvalues. An approximate solution of this equation is given in the next section. It can be shown from the asymptotic formula (4.8) that an alternative statement of (5.13) is

$$
\left.\begin{array}{rl}
d \phi\left(\frac{1}{2} a\right) / d x=0 & (\phi \text { symmetric }) \\
\phi\left(\frac{1}{2} a\right)=0 & (\phi \text { antisymmetric })
\end{array}\right\} .
$$

The argument may be repeated for $x \geqq \frac{1}{2} a$. Thus it has been proved that

where

$$
f(x)=\Phi(x)+\delta(x)
$$

and

$$
\begin{aligned}
\Phi(x) & =\phi(x) \quad\left(0 \leqq x \leqq \frac{1}{2} a\right), \\
& = \pm \phi(a-x)\left(\frac{1}{2} a \leqq x \leqq a\right),
\end{aligned}
$$

$$
\int_{0}^{a} \delta^{2}(x) d x=O\left(e^{-a \tau_{1}}\right)
$$

A multiplicative constant has been dropped from the solution so that $f$ may satisfy (1.3).

It will now be proved that $\delta$ is uniformly small. To do this (5.15) is substituted into (1.1). Then

$$
f(x)=\lambda \int_{0}^{a} k(x-t) \Phi(t) d t+O\left(e^{-\frac{1}{2} a \tau_{1}}\right)
$$

by Schwartz's inequality and (5.16). Now using the fact that $\phi$ is the solution of (4.1),

$f(x)=\phi(x)-\lambda \int_{\frac{1}{2} a}^{\frac{3}{4} a} k(x-t)[\phi(t) \mp \phi(a-t)] d t-\lambda \int_{\frac{3}{4} a}^{\infty} k(x-t) \phi(t) d t$

$$
\pm \lambda \int_{\frac{3}{4} a}^{a} k(x-t) \phi(a-t) d t+O\left(e^{-\frac{1}{2} a \tau_{1}}\right)
$$


Schwartz's inequality then shows from the known asymptotic form (4.8) of $\phi$ and from (5.14) that the first integral is small. By splitting the integrand into the form $[k(x-t) \exp (t \tau)][\phi(t) \exp (-t \tau)]$ and using Schwartz's inequality the second integral is seen to be small, a similar argument applying for the last term. The argument is similar for $\frac{1}{2} a \leqq x \leqq a$. Then

$$
\delta(x)=O\left(e^{-\frac{1}{2} a \tau_{1}}\right),
$$

for sufficiently large $a$.

\section{Further approximations}

In Section 5 the approximate solution of (1.1) was obtained in terms of the Wiener-Hopf solution. The Wiener-Hopf solution may be expressed in quadratures (9). However, the evaluation of the integrals is sometimes difficult and it is therefore useful to approximate still further to the solution by using the asymptotic formula (4.8) and to find approximate solutions of (5.13). Certain general characteristics of the solution then become clear. These approximations are carried out in this section where it is also proved that each of the eigenvalues is of index unity. A general discussion of the results is then given in the next section.

Approximate solutions of (5.13) are first obtained. From (4.5) we may define $K_{+}(\mu ; \mu) / K_{+}(-\mu ; \mu)=-\exp [i \theta(\mu)]$ where $\theta(0)=\pi$. Also as $K_{+}(\mu ; \mu)$ is a continuous differentiable function of $\mu, \theta$ may be defined to be so also. (5.13) may then be written as

$$
\mu a=n \pi+\theta(\mu)+h(a, \mu),
$$

where $n$ is any integer such that $c a^{-1}<\mu<\mu_{1}$, and even and odd $n$ give symmetric and antisymmetric eigenfunctions respectively. Also $h$ and $\partial h / \partial \mu$ are both $O\left(a^{\frac{3}{2}} \exp \left(-a \tau_{1}\right)\right)$. From the differentiability of $\theta$ and $h$ it is easy to show by the mean value theorem that successive solutions of (5.1) are such that

$$
\mu_{r+1}-\mu_{r}=\pi a^{-1}+O\left(a^{-2}\right) .
$$

As $\theta(0)=\pi$ the first eigenvalue must be such that

$$
\mu_{0}=\pi a^{-1}+O\left(a^{-2}\right) \text {. }
$$

(There may possibly be one smaller solution of (6.1) but this will not satisfy (d) of Section 3.) Thus for $\mu$ in the given range

$$
\mu_{n}=(n+1) \pi a^{-1}\left[1+O\left(a^{-1}\right)\right] \quad(n=0,1 \ldots) \text {. }
$$

The eigenvalues $\lambda_{n}$ are then found from the relation $\lambda_{n}=K^{-1}\left(\mu_{n}\right)$. Better approximations than (6.4) may be obtained by evaluating $\theta(\mu)$ approximately. Thus Widom (12) obtains (by a different method) an approximation which holds for small $\mu$. This result may also be obtained without difficulty by the present method.

It is now easy to show that each eigenvalue is of index unity. For it was proved ((c) of Section 3) that if there are two symmetric eigenfunctions there 
must be an antisymmetric eigenfunction. But from (6.2) these two types cannot occur for the same eigenvalue. Therefore the index of each eigenvalue is unity.

From the asymptotic formula (4.8) and from (5.15) it is obvious that

$$
f_{n}(x)=\cos \left[\mu_{n}\left(x-\frac{1}{2} a\right)+\frac{1}{2} n \pi\right]+O\left[e^{-\frac{1}{2} t_{1} x}+e^{-\frac{1}{2} \tau_{1}(a-x)}\right] \quad(n=0,1, \ldots), \ldots
$$
where the $\mu_{n}$ are given by (6.1) or approximately by (6.4).

\section{Discussion of results. Analogy with the Sturm-Liouville system}

The known results about the spectrum of this type of integral equation are not complete. As stated in the introduction Widom (12) obtained a relation (1.5) which is however only valid for small $\mu$. Grenander and Szegö (5) obtained a result for very general kernels concerning the average number of eigenvalues in a given interval for large $a$. They proved that

$$
\lim _{a \rightarrow \infty} \pi a^{-1}\left\{\text { Number of values of } \lambda_{n}^{-1} \text { in }(C, D)\right\}=m(C \leqq K(x) \leqq D),
$$

where $m$ denotes measure. In the present investigation more restrictive assumptions are made about the kernel. And under these conditions more accurate predictions may be made. For example, in constrast with Widom's result (1.5) the present results are valid for any given range $0<\mu<\mu_{1}$ for choice of $a$ (dependent only on $\mu_{1}$ ) large enough.

Also the results when conditions $B$ and $C$ hold resemble the results of Sturm-Liouville theory (in fact the result about the oscillations is only a partial one); for, the index of each eigenvalue is unity. Also it may be seen from (6.5) and (6.2) that the solutions are oscillatory (and indeed trigonometric) and the zeros of successive solutions interlace in the range where the error in (6.5) is small; the only part of the range where the error is not small is the relatively small range near the endpoints. It would be theoretically possible from a more exact examination of the Wiener-Hopf solution to find out whether this is so even near the end points. However, it has not yet been found possible to do this for general kernels.

As stated in the introduction Carrier (1) made a suggestion about the possibility of expressing the solution of (1.1) in terms of the Wiener-Hopf solution. This has been shown to be true if conditions $B$ and $C$ hold.

However, if condition $B$ is removed, then for some value of $\lambda$, the equation $1-\lambda K(w)=0$ may have $2 r(r>1)$ real roots. In this case an equation of the form (1.1) may be constructed with some eigenvalues of index greater than one. Probably the situation is then that the index of an eigenvalue is not greater than the index of the eigenvalue of the corresponding Wiener-Hopf solution. This case could be examined by an extension of the present method.

If condition $C$ is removed and $d^{2} K(0) / d w^{2}=0$, then $1-\lambda K(w)=0$ will have complex roots which approach the real axis as $\mu$ approaches zero. In this case the approximate solution (5.15) and (6.1) will hold for $0<\mu_{2}<\mu<\mu_{1}$ (fixed $\mu_{1}$ and $\mu_{2}$ ). But the proof given will not hold for the original range $0<\mu<\mu_{1}$. 


\section{The solution of equation (1.2)}

The solution of (1.2) may be obtained by a method very similar to that used in Section 5. From the definition of the kernel, it is obvious that it is regular (3) and therefore (1.2) has one and only one solution for $\lambda<\lambda_{0}$ the smallest eigenvalue. The solution will be given approximately for $\lambda<K^{-1}(0)$.

The solution may be given in terms of $H(x, t)$ the resolvent kernel of the corresponding Wiener-Hopf equation (4.2). An expression for $H$ when the kernel is of a certain type is given in (6). It is found that

where

$$
\left.\begin{array}{rlr}
f(x) & =g(x)+\int_{0}^{a} H(x, t) g(t) d t+\delta(x) & \left(0 \leqq x \leqq \frac{1}{2} a\right) \\
& =g(x)+\int_{0}^{a} H(a-x, a-t) g(t) d t+\delta(x) & \left(\frac{1}{2} a \leqq x \leqq a\right)
\end{array}\right\}
$$

$$
\delta(x)=O\left[e^{-\frac{1}{2} a \sigma}\left(1-\lambda \lambda_{0}^{-1}\right)^{-1}\left\{\int_{0}^{a} g^{2}(t) d t\right\}^{\frac{1}{1}}\right],
$$

for sufficiently large $a$ and $\lambda<K^{-1}(0)$, where (e) of Section 3 has been used. The smallest eigenvalue $\lambda_{0}$ is given approximately by (6.4).

Alternatively this may be expressed by saying that $f$ is approximately the solution of (4.2) given by (4.9) for $0 \leqq x \leqq \frac{1}{2} a$, with a similar result applying for $\frac{1}{2} a \leqq x \leqq a$.

\section{Appendix 1}

Bounds will now be obtained for $R(w)$ and $S(w)$ which appear in equations (5.5) and (5.6). Three inequalities (A1), (A2) and (A3) are needed in the proof. The proofs are given for $d k / d w$ a continuous function, the extension to the case when it has a finite number of jump discontinuities being done by splitting the interval into subintervals. $C$ stands for any constant (not necessarily always the same) independent of $a$.

Lemma 1.

$$
\begin{aligned}
& |f(x)| \leqq \lambda a^{\frac{1}{2}} C . \\
& \left|f^{\prime}(x)\right| \leqq \lambda a^{\frac{1}{2}} C\left\{\int_{0}^{a}\left[\frac{d k(x-t)}{d t}\right]^{2} d t\right\}^{\frac{1}{2}} . \\
& \left|F_{+}(w)\right| \leqq \lambda a^{\frac{1}{2}} C|w|^{-1} \exp \{-a \mathfrak{I} w\} \quad(\mathfrak{I} w>0) .
\end{aligned}
$$

Proof. As $k$ and $d k / d u$ belongs to $L^{2}(0, \infty)$ the first two results are obvious on application of Schwartz's inequality.

To prove (A3) integrate the expression for $F_{+}$by parts and use Schwartz's inequality. Then

$$
\left|F_{+}(w)+\frac{f(a) e^{i a w}}{i w}\right| \leqq e^{-a \Im w}|w|^{-1}\left\{\int_{a}^{\infty}[d f / d x]^{2} d x\right\}^{\frac{1}{2}}
$$


After use of (A2) and changes of variable,

$$
\left|F_{+}(w)+\frac{f(a) e^{i a w}}{i w}\right| \leqq C e^{-a 9 w}|w|^{-1} \lambda a^{\frac{1}{2}}\left\{\int_{0}^{\infty} d x \int_{x}^{\infty}\left[\frac{d k(u)}{d u}\right]^{2} d u\right\}^{\frac{1}{2}} .
$$

The result now follows on using (A1) and condition $A$ of Section 2.

The remainder function $R(w)=S(-w)$ is defined by Latter as

$$
R(w)=\frac{1}{2 \pi i} \int_{i \tau_{1}-\infty}^{i \tau_{1}+\infty} \frac{F_{+}\left(w^{\prime}\right) d w^{\prime}}{\left(w^{\prime}-\mu\right) K_{-}\left(w^{\prime}\right)\left(w^{\prime}-w\right)} .
$$

Lemma 2. The remainder function $R(w)$ satisfies the condition

$$
|R(w)| \leqq C a^{\frac{1}{2}} \lambda e^{-a \tau_{1}}(1+|w|)^{-1} \log (1+|w|) \quad\left(\mathfrak{J}_{w} \leqq \tau_{2}\right),
$$

with a similar result for $S(w)$.

Proof. From the factorisation theorem it is known that $\left|w K_{-}(w)\right|$ lies between positive bounds, and $K_{-}(w)$ is regular along the line of integration. Therefore from (A3),

$$
|R(w)| \leqq C \lambda a^{\frac{1}{2}} e^{-a \tau_{1}} \int_{i \tau_{1}-\infty}^{i \tau_{1}+\infty} \frac{d w^{\prime}}{\left|w^{\prime}\right|\left|w^{\prime}-w\right|} \quad\left(\Im w<\tau_{2}<\tau_{1}\right) .
$$

The result follows immediately from the obvious bounds for the integral.

\section{Appendix 2}

In Section 5 it was proved that every eigenfunction of (1.1) must be given by (5.15) and the relation between $\mu$ and $a$ by (5.13), for $\mu$ lying in a given range $0<\mu<\mu_{1}$ and for sufficiently large $a$. The converse will now be proved. That is, every $\mu$ given by (6.1) provides an eigenfunction of (1.1). The argument will be by contradiction.

In Section 5 it was shown that

$$
\Phi_{N}(x)=\lambda_{N} \int_{0}^{a} k(x-t) \Phi_{N}(t) d t+\delta(x)
$$

where

$$
\int_{0}^{a} \delta^{2}(x) d x=O\left(e^{-\frac{1}{2} a \tau_{1}}\right),
$$

and $\Phi_{N}$ is determined by (6.1). Suppose that some $\Phi$ say $\Phi_{N}$ is not an approximate eigenfunction. Then by Mercer's theorem (11) the sum of reciprocals of the eigenvalues is absolutely convergent and the solution of (A6) is given by the absolutely and uniformly convergent series

$$
\Phi_{N}(x)=\delta(x)+\lambda_{N} a^{-1} \sum_{n=0}^{\infty} \frac{f_{n}(x)}{\lambda_{N}-\lambda_{n}} \int_{0}^{a} f_{n}(t) \delta(t) d t,
$$


where $f_{n}$ are the solutions of (1.1). Then

$$
\int_{0}^{a} \Phi_{N}^{2}(x) d x=O\left\{a^{\frac{1}{2}} e^{-\frac{1}{2} a \tau_{1}}\left[1+\lambda_{N} \sum_{n=0}^{\infty}\left|\lambda_{N}-\lambda_{n}\right|^{-1}\right]\right\}
$$

after multiplication by $\Phi_{N}$ and integration and use of Schwartz's inequality together with (A7).

Now choose an eigenvalue $\lambda_{N_{1}}>2 \lambda_{N}$ and choose $a$ so large that the approximation holds for $\lambda<\lambda_{N_{1}}$. Then all the eigenvalues in the first part of the sum must satisfy (6.1) although it is possible that some solutions, in this case $\lambda_{N}$ is one of them, may not provide eigenfunctions. Therefore

$$
\begin{aligned}
\sum_{\substack{n=1 \\
n \neq N}}^{N_{1}}\left|\lambda_{N}-\lambda_{n}\right|^{-1} & \leqq N_{1} \sup \left|\lambda_{N}-\lambda_{n}\right|^{-1} \\
& \leqq \mu_{N_{1}} a \pi^{-1} \sup \left|\lambda_{N}-\lambda_{n}\right|^{-1}
\end{aligned}
$$

But the least value of $\left|\lambda_{N}-\lambda_{n}\right|$ is taken when $n=N \pm 1$. Suppose it is $N+1$ (the same proof will apply for $N-1$ ). Then using the relation between $\lambda$ and $\mu$, and (6.2), and the mean value theorem

$$
\left|\lambda_{N}-\lambda_{n}\right| \geqq \lambda_{N} \lambda_{N+1}\left[\pi a^{-1}+O\left({ }^{-2}\right)\right] d K\left(\mu_{N}+\xi \pi a^{-1}\right) / d w \quad(0 \leqq \xi<1) .
$$

But $d K / d w$ is by definition non-zero except at $w=0$, and near this point $d K / d w \sim-B w$ (as $d^{2} K(0) / d w^{2}=0$ ) for some $B>0$. The lower bound of $|d K / d w|$ in the interval $\left(\pi / 2 a, \mu_{1}\right)$ may therefore for large enough $a$ be taken to be $\pi B / 2 a$. Then

Also

$$
\sum_{\substack{n=1 \\ n \neq N}}^{N_{1}}\left|\lambda_{N}-\lambda_{n}\right|^{-1}=O\left(a^{3}\right)
$$

$$
\sum_{n=N_{1}}^{\infty}\left|\lambda_{N}-\lambda_{n}\right|^{-1} \leqq 2 \sum_{n=N_{1}}^{\infty} \lambda_{n}^{-1}
$$

From (A9) and (A10)

$$
\leqq 2 a k(0) \text {. }
$$

$$
\int_{0}^{a} \Phi_{N}^{2}(t) d t=O\left(a^{4} e^{-\frac{1}{2} a \tau_{1}}\right)
$$

which for choice of sufficiently large $a$ contradicts (1.3). This completes the proof.

\section{REFERENCES}

(1) G. F. CARRIER, 50 Jahre Grenzschichtforschung (Edited by H. Görtler and W. Tollmien) (Vieweg: Braunschweig, 1955), 13-20.

(2) K. M. CASE, Ann. Phys., 2 (1957), 384-405.

(3) R. Courant and D. Hilbert, Methods of Mathematical Physics, Vol. 1 (Interscience: New York, 1953). 
(4) V. FocK, C.R. Acad. Sci. U.R.S.S., 36 (1942), 133-136.

(5) U. Grenander and G. Szegö, Toeplitz Forms and their Applications (Univversity of California Press, 1958).

(6) M. G. KreIN, Uspekhi Math. Nauk., 13 (1958), 8, 5 (83), 3-120.

(7) R. LATter, Quart. App. Math., 16 (1958), 21-31.

(8) B. Noble, The Wiener-Hopf Technique (Pergammon Press, London, 1958).

(9) E. PAley and N. WIENer Fourier Transforms (Amer. Math. Soc. Colloquium Publication 19, New York, 1934). 1948).

(10) E. C. TIтchmaRsh, The Theory of Fourier Integrals (Clarendon Press, Oxford,

(11) F. G. TRICOMI, Integral Equations (Interscience, New York, 1957).

(12) H. Widom, Trans. Amer. Math. Soc., 88 (1958), $491-522$.

(13) H. Widom, Trans. Amer. Math. Soc., 100 (1961), 252-262.

Department of Applied Mathematics

THE UNIVERSITY

SHEFFIELD, 10 